\title{
MINLP Model for the Synthesis of Heat Exchanger Networks with Handling Pressure of Process Streams
}

\author{
Viviani C. Onishi ${ }^{\mathrm{a}, \mathrm{b}, \mathrm{c}, *}$, Mauro A. S. S. Ravagnani ${ }^{\mathrm{a}}$, José A. Caballero ${ }^{\mathrm{b}}$ \\ ${ }^{a}$ Department of Chemical Engineering, State University of Maringá, Av. Colombo 5790, \\ Maringá-PR 87020-900, Brazil
}

\begin{abstract}
This paper introduces a new mathematical model for the simultaneous synthesis of heat exchanger networks (HENs), wherein the handling pressure of process streams is used to enhance the heat integration. The proposed approach combines generalized disjunctive programming (GDP) and mixed-integer nonlinear programming (MINLP) formulation, in order to minimize the total annualized cost composed by operational and capital expenses. A multi-stage superstructure is developed for the HEN synthesis, assuming constant heat capacity flow rates and isothermal mixing, and allowing for streams splits. In this model, the pressure and temperature of streams must be treated as optimization variables, increasing further the complexity and difficulty to solve the problem. In addition, the model allows for coupling of compressors and turbines to save energy. A case study is performed to verify the accuracy of the proposed model. In this example, the optimal integration between the heat and work decreases the need for thermal utilities in the HEN design. As a result, the total annualized cost is also reduced due to the decrease in the operational expenses related to the heating and cooling of the streams.
\end{abstract}

Keywords: optimization, mixed-integer nonlinear programming (MINLP), heat exchanger network (HEN), heat integration, handling pressure.

\section{Introduction}

Optimizing energy use through the application of more efficient processing technologies is essential to improve the energy conservation in industrial processes (Onishi et al., 2013). The increasing global energy demand allied with the current high cost of energy, and the tightening environmental regulations on gaseous emissions are among the many driving forces behind the need for energy conservation (Razib et al., 2012) and efficiency in processing plants. The synthesis of heat exchanger networks (HENs) has been extensively studied over the past few decades (Huang and Karimi, 2012), due to the importance of thermal integration in the reduction of energy consumption and efficient energy usage (Ravagnani and Silva, 2012). Amongst the major research areas, mathematical programming stands out for treating the HEN design as an optimization problem, in order to obtain an optimal network in economic and thermodynamic terms. Although the simultaneous methods are, generally, more difficult to implement and solve, it can lead to larger economic advantages (Kamath et al., 2012).

Handling pressure is extremely important in many industrial plants, such as oil refineries and cryogenic processes, due to being responsible for large energy consumption. Despite the numerous efforts to solve the problem of HEN synthesis, few studies related to processes optimization involving pressure manipulation and heat 
integration of streams are available in the literature. Aspelund et al. (2007) proposed an approach based on Extended Pinch Analysis and Design (ExPAnD) to study the expansion of streams at sub-ambient conditions. In their work, only a graphical interpretation of pressure exergy are considered to minimize energy requirements of the system. Wechsung et al.(2011) presented a model for the HEN synthesis with streams that are subject to compression and expansion. The authors combine pinch analysis, exergy analysis and mathematical programming in a formulation to minimize the total irreversibility of the process. However, the costs involved in the network design as well as the possibility of coupling equipment are not evaluated, only the aspects related to heuristics and exergy analisys are taken into account during the HEN synthesis.

This paper introduces a new mathematical model for the simultaneous HENs synthesis, wherein the handling pressure of process streams is used to enhance the heat integration. The proposed model combines generalized disjunctive programming (GDP) and mixedinteger nonlinear programming (MINLP) formulation, in order to minimize the total annualized cost composed by operational and capital expenses. The superstructure is based on the model of Yee and Grossmann (1990), allowing for stream splits and assuming constant heat capacity flow rates and isothermal mixing. However, the streams pressure and temperature should be treated as optimization variables. In addition, the model allows for coupling of compressors and turbines, increasing further its complexity. A case study is performed to verify the accuracy of the proposed model. The results indicate that optimal integration between the heat and work decreases the total annualized cost, due to reduced need for thermal utilities in the HEN design.

\section{Problem statement}

Given a set of hot and cold process streams with a known supply state-temperature and pressure - and a target state in which some streams have pressures that differ from the inlet conditions, energy supplies for heating and cooling, and pressure manipulation equipment, with their respective costs. The main objective of the model is to synthetize an optimal HEN with handling pressure of streams that minimizes the total annualized cost, considering the operational expenses and the capital investment in the various units of the network. In the proposed approach, some flows should follow a specific route of expansion and compression via turbines and compressors. This route is selected based on the work of Wechsung et al. (2011), wherein the "plus-minus" principle is used to identify the best way of pressure manipulation of streams so that the energy requirements may be reduced. Thus, considering a maximum of three pressure manipulations, the hot streams can potentially be cooled, compressed, cooled, expanded, heated, compressed and cooled. Similarly, the cold streams can be heated, expanded, heated, compressed, cooled, expanded and heated. The processes of streams expansion and compression are formulated as an isentropic process, through the introduction of a constant efficiency factor to model real processes. In addition, the proposed model allows for coupling of turbines and compressors as long as the costbenefit ratio is respected in order to save energy. This fact, added to the high nonlinearity and non-convexity of the cost correlations, confer an even higher degree of complexity on the model. For simplification, all streams should behave as ideal gases.

\section{MINLP-based model}

The proposed model is developed in generalized disjunctive programming (GDP) and mixed integer non-linear programming (MINLP) formulation. The mathematical model 
is based on the MINLP model for HEN design presented by Yee and Grossmann (1990), assuming isothermal mixing and constant heat capacity flow rates, and allowing for streams splits. The superstructure is represented by stages according to temperatures. In each of these stages, a hot stream can exchange heat with all cold streams, and vice versa. The number of stages is equal to the maximum number of possible heat exchanges between hot and cold streams. Moreover, heaters and coolers are allocated in the ends of the streams.

The main difference between the proposed model and the Yee and Grossmann (1990) superstructure is that, in this model, the streams are subjected to handling pressure, and they must be connected to the HEN through turbines and compressors. Consequently, the outlet temperatures of the HEN should correspond to the temperatures of the inlet streams in the respective pressure equipment. Thus, the process conditions (i.e., stream temperature and pressure) are considered as unknown variables that must be optimized in order to obtain an optimal design with a minimal cost. In consequence, this proposed approach is significantly more complex than the standard problem of heat integration as formulated by Yee and Grossmann (1990). In fact, the streams can temporarily change their identity; as a result, a hot stream can behave as a cold stream and, analogously, a cold stream can behave like a hot stream. Furthermore, some process streams can operate as thermal utilities, mitigating problems related to excess or deficit of energy in the system. Therefore, there is no clear distinction among hot and cold streams, nor between thermal utilities and streams. Moreover, the heat exchange among parts of the same stream that assume other identity, like as the placement of thermal utilities between stages of expansion and compression should be forbidden, through the implementation of constraints in the model that increases further the difficult to solve it. As the conventional problem of HENs synthesis is extended to include streams subjected to handling pressure, a operator for pressure manipulation and a GDP-based operator for the coupling of compressors and turbines are added in the formulation:

$$
\left[\begin{array}{c}
y_{c, e} \\
W C_{c}=W E_{e} \\
C_{\text {electricity }}=C E \cdot W C_{c}=0
\end{array}\right]
$$

Using the big-M reformulation, the disjunction may be expressed as follows:

$$
\begin{aligned}
& W C_{c}-W E_{e} \leq M_{1}\left(1-y_{c, e}\right) \\
& W C_{c}-W E_{e} \geq-M_{1}\left(1-y_{c, e}\right) \\
& C_{\text {electricity }} \leq M_{2}\left(1-y_{c, e}\right) \\
& C_{\text {electricity }} \geq C E \cdot W C_{c}-M_{2} \cdot y_{c, e}
\end{aligned}
$$

In which $M$ is a positive parameter that is large enough to validate the formulation (1). The parameter $M_{l}$ is calculated as the difference between the upper bound of the expansion work and the lower bound of the compression work, and the parameter $M_{2}$ is calculated as the difference between the upper and lower bounds of the electricity cost.

The superstructure is written in GAMS and solved with the SBB solver. As the model is highly nonlinear and non-convex, it is very difficult to solve it to global optimality with the available global solvers due the large CPU time required. Nevertheless, the use of a simple branch and bound based solver, such as the SBB solver, allows obtaining a 
solution near the global optimum. To solve the model, one must impose limits (i.e., upper and lower bounds) on all variables, and provide initial values with physical meaning.

\section{Case study}

A case study is performed to verify the applicability of the developed MINLP model, for obtaining an optimal HEN synthesis with handling pressure of streams at subambient conditions. The example was extracted from Wechsung et al. (2011), considering a two-fold flow in all streams. One hot stream $\mathrm{H} 1$ and one cold stream $\mathrm{C} 1$ are at constant pressure, whereas a second cold stream $\mathrm{C} 2$ is expanded from 0.4 to 0.1 $\mathrm{MPa}$. The pressure manipulation route for $\mathrm{C} 2$ includes the stages of expansion, compression and expansion, and requires heat integration in the HEN between each of these stages. Thus, $\mathrm{C} 2$ behaves as $\mathrm{C} 3$ after the first expansion, as $\mathrm{H} 2$ after compression, and finally, as $\mathrm{C} 4$ after the last expansion. The heat capacity and flow rates of all streams are known constants. The problem data are presented in Table 1. A superstructure with four stages, and the possibility of stream splits is considered for the HEN synthesis. The unknown inlet temperatures can vary between 103-373 K, the pressure of the $\mathrm{C} 3$ stream is restricted to $0.1-0.4 \mathrm{MPa}$, and the pressure of the $\mathrm{H} 2$ stream is restricted to $0.1-0.6 \mathrm{MPa}$. The individual heat transfer coefficients for all streams are maintained at $0.1 \mathrm{~kW} / \mathrm{m}^{2} \mathrm{~K}$, and a hot and cold utility coefficient of 1.0 $\mathrm{kW} / \mathrm{m}^{2} \mathrm{~K}$ is considered. An annualized capital cost factor of $f=0.18$ (10\% interest rate over 8 years) is assumed. In addition, $T^{h}{ }_{U}=383 \mathrm{~K}, T_{U}^{c}=93 \mathrm{~K}$, and $\Delta T_{\min }=4 \mathrm{~K}$. The HEN synthesis considers the possibility of coupling equipment with an efficiency of $\eta=$ 0.7 and $\kappa=1.51$ for both the turbine and the compressor.

Table 1. Problem data for the case study.

\begin{tabular}{ccccc}
\hline Stream & $F_{s} C p_{s}(\mathrm{~kW} / \mathrm{K})$ & $T_{s, i n}(\mathrm{~K})$ & $T_{s, \text { out }}(\mathrm{K})$ & $p_{s}(\mathrm{MPa})$ \\
\hline H1 & 6.0 & 288 & 123 & 0.1 \\
C1 & 4.0 & 213 & 288 & 0.1 \\
C2 & 3.4 & 113 & - & 0.4 \\
C3 & 3.4 & - & - & - \\
H2 & 3.4 & - & - & - \\
C4 & 3.4 & - & 288 & 0.1 \\
\hline
\end{tabular}

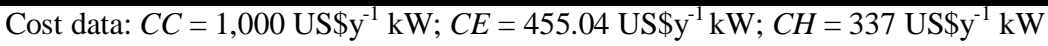

Firstly, the HEN is designed without handling pressure of stream $\mathrm{C} 2$, i.e., all streams are at constant pressure. The optimal HEN is obtained with two heat exchangers $(A=42.47$ $\mathrm{m}^{2}, Q=77.87 \mathrm{~kW}$; and $\left.A=95.88 \mathrm{~m}^{2}, Q=371,64 \mathrm{~kW}\right)$, two heaters with areas of $A=$ $20.33 \mathrm{~m}^{2}(Q=222.14 \mathrm{~kW})$, and $A=19.73 \mathrm{~m}^{2}(Q=223.36 \mathrm{~kW})$ located at the ends of streams $\mathrm{C} 1$ and $\mathrm{C} 2$, and one cooler placed at the end of $\mathrm{H} 1\left(A=102.48 \mathrm{~m}^{2}, Q=540.50\right.$ $\mathrm{kW})$. In this case, no compression and/or expansion work is produced in the network. The total annualized cost of the HEN with this configuration is $866,171 \mathrm{USS}^{-1}{ }^{1}$, in

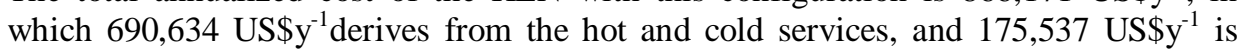
related to the capital investment in equipment. Secondly, the proposed MINLP model is used to solve the problem. The results indicate that the optimal HEN is obtained with four heat exchangers $\left(A=90.39 \mathrm{~m}^{2}, Q=171.61 \mathrm{~kW} ; A=287.51 \mathrm{~m}^{2}, Q=506.47 \mathrm{~kW} ; A\right.$ $=134.79 \mathrm{~m}^{2}, Q=179.86 \mathrm{~kW}$; and $\left.A=176.94 \mathrm{~m}^{2}, Q=300 \mathrm{~kW}\right)$, one cooler located in the end of $\mathrm{H} 1\left(A=25.57 \mathrm{~m}^{2}, Q=132.07 \mathrm{~kW}\right)$, and one heater with area of $7.74 \mathrm{~m}^{2}(Q=$ $141.99 \mathrm{~kW})$ placed at the end of stream $\mathrm{C} 4$. In this second case, two turbines and one 
compressor are also required, in which the expander EX1 is coupled to CO1 (WC $=W E$ $=108.13 \mathrm{~kW}$ ); therefore, the cost of electricity is zero. The expansion work produced by EX2 is equal to $104.92 \mathrm{~kW}$. The results obtained for the decision variables and optimal HEN configuration are presented in Figure 1.

The total annualized cost of the HEN with this configuration is $603,844 \mathrm{USS}^{-1}$ (composed by $C_{\text {operational }}=179,924 \mathrm{US}^{\mathrm{y}} \mathrm{y}^{-1}$ and $\left.C_{\text {capital }}=423,920 \mathrm{US}^{\mathrm{y}} \mathrm{y}^{-1}\right)$, which represents $30 \%$ of savings in the total annualized cost of the HEN over that obtained in the first case, i.e., without pressure manipulation of the stream C2. The mathematical model has 322 continuous variables, 25 discrete variables, and 460 constraints with 1,411 Jacobian elements (non-zeros), of which 200 are nonlinear. The CPU time is $57 \mathrm{~s}$ with the SBB solver under the software GAMS (version 24.0.2). The problem was solved using a personal computer with an Intel Core 2 Duo $2.40 \mathrm{GHz}$ processor and 3.00 GB RAM running Windows 7 Ultimate.

\section{Conclusions}

A new MINLP model for HEN synthesis with handling pressure of streams is proposed to optimize the integration between heat and work. The developed approach, involves generalized disjunctive programming (GDP) and mixed-integer nonlinear programming (MINLP) formulation. The conventional HEN synthesis problem is expanded to harness energy from streams that undergo pressure manipulation. As a result, intermediate pressures and temperatures are treated as unknown variables that must be optimized. The streams subjected to handling pressure should be connected to the HEN via compressors and turbines. The hot and/or cold streams should follow a specific pressure manipulation route to reduce the energy requirements. The possibility of coupled equipment is studied to optimize the HEN design by minimizing the total annualized cost, composed by operational and capital expenses of the network.

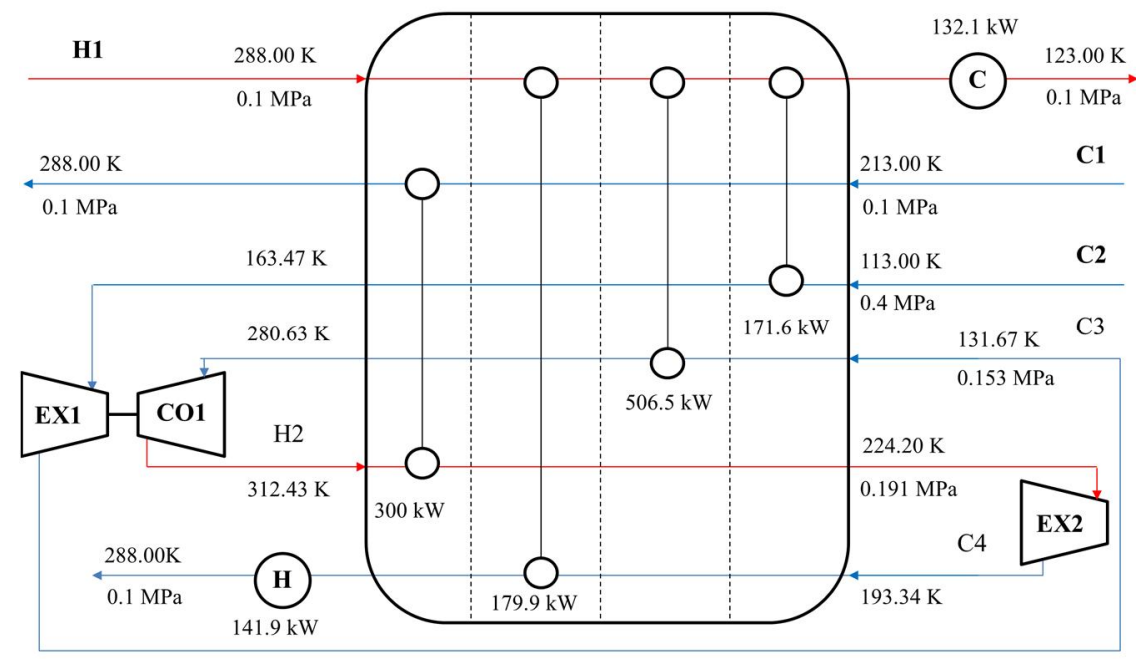

Figure 1. Optimal HEN configuration obtained for the case study.

The results indicate that the handling pressure of streams enhances the heat integration, decreasing significantly the amount of necessary thermal utilities in the HEN design. Consequently, the total annualized cost is reduced in $30 \%$ due the diminution of 
operational expenses related to heating and cooling of process flows. The total annualized cost also decreases in $8 \%$ when compressors and turbines are coupled, to allow the expansion work to satisfy the energy requirement of the compressors. In this case, the operational expenses associated to electricity are zero. In the example, up to three pressure manipulations of streams are allowed. However, a larger number of pressure changes can easily be implemented in the model through the use of a larger quantity of compressors and turbines in each stream subjected to pressure manipulation.

\section{Nomenclature}

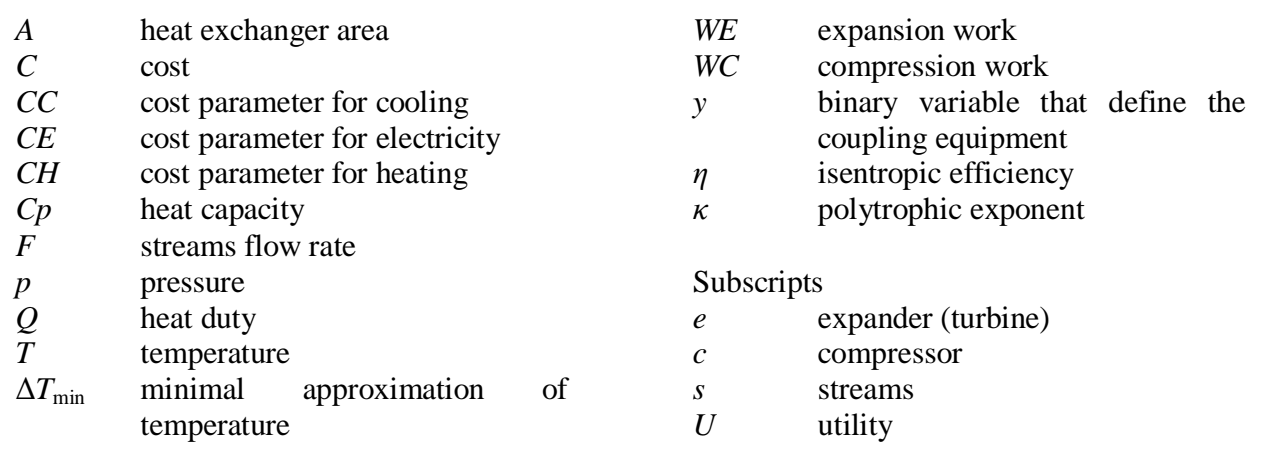

\section{Acknowledgments}

This study was financed by the Brazilian agency "Coordenação de Aperfeiçoamento de Pessoal de Nível Superior - CAPES” (process n¹0758/12-7), and the Spanish Ministry of Science and Innovation and Ministry of Economy and Competitiveness (project CTQ2012-37039-C02-02).

Affiliation of José A. Caballero is Department of Chemical Engineering, University of Alicante, Ap Correos 99, Alicante03080, Spain. Viviani C. Onishi also has the following affiliation: CAPES Foundation, Ministry of Education of Brazil, Brasília-DF 7004020, Brazil.pg51551@uem.br/viviani.onishi@hotmail.com

\section{References}

A. Aspelund, D.O. Berstad, T. Gundersen, 2007, An extended pinch analysis and design procedure utilizing pressure based exergy for subambient cooling, Applied Thermal Engineering, 27, 2633-2649.

A. Wechsung, A. Aspelund, T. Gundersen, P.I. Barton, 2011, Synthesis of heat exchanger networks at subambient conditions with compression and expansion of process streams, Process Systems Engineering, 57, 8, 2090-2108.

K.F. Huang, I.A. Karimi, 2012, Heat exchanger network synthesis using a hyperstructure of stagewise stream superstructures, Computer Aided Chemical Engineering, 31, 1552-1556.

M.A.A.S. Ravagnani, A.P. Silva, 2012, Retrofit of heat exchanger networks including the detailed equipment design, Computer Aided Chemical Engineering, 31, 235-239.

M.S. Razib, M.M.F. Hasan, I.A. Karimi, 2012, Preliminary synthesis of work exchange networks, Computers and Chemical Engineering, 37, 262-277.

R.S. Kamath, L.T. Biegler, I.E. Grossmann, 2012, Modeling multistream heat exchangers with and without phase changes for simultaneous optimization and heat integration, AIChE Journal, 58, 190-204.

T.F. Yee, I.E.Grossmann, 1990, Simultaneous optimization models for heat integration. II. Heat exchanger network synthesis, Computers and Chemical Engineering, 14, 10, 1165-1184.

V.C. Onishi, M.A.S.S. Ravagnani, J.A. Caballero, 2013, Mathematical programming model for heat exchanger design through optimization of partial objectives, Energy Conversion and Management, 74, 60-69. 